\title{
Surface Plasmons and Surface Enhanced Raman Spectra of Aggregated and Alloyed Gold-Silver Nanoparticles
}

\author{
Y. Fleger ${ }^{1,2}$ and M. Rosenbluh"1,2 \\ ${ }^{1}$ Department of Physics, The Jack and Pearl Resnick Institute for Advanced Technology, Bar-Ilan University, 52900 Ramat-Gan, Israel \\ ${ }^{2}$ Institute of Nanotechnology and Advanced Materials, Bar-Ilan University, 52900 Ramat-Gan, Israel
}

Correspondence should be addressed to Y. Fleger, gutmany@mail.biu.ac.il

Received 17 February 2009; Accepted 10 May 2009

Recommended by Roberto Morandotti

Effects of size, morphology, and composition of gold and silver nanoparticles on surface plasmon resonance (SPR) and surface enhanced Raman spectroscopy (SERS) are studied with the purpose of optimizing SERS substrates. Various gold and silver films made by evaporation and subsequent annealing give different morphologies and compositions of nanoparticles and thus different position of the SPR peak. SERS measurements of 4-mercaptobenzoic acid obtained from these films reveal that the proximity of the SPR peak to the exciting laser wavelength is not the only factor leading to the highest Raman enhancement. Silver nanoparticles evaporated on top of larger gold nanoparticles show higher SERS than gold-silver alloyed nanoparticles, in spite of the fact that the SPR peak of alloyed nanoparticles is narrower and closer to the excitation wavelength. The highest Raman enhancement was obtained for substrates with a two-peak particle size distribution for excitation wavelengths close to the SPR.

Copyright (C) 2009 Y. Fleger and M. Rosenbluh. This is an open access article distributed under the Creative Commons Attribution License, which permits unrestricted use, distribution, and reproduction in any medium, provided the original work is properly cited.

\section{Introduction}

Since the discovery of SERS many variations of the technique have been explored using different substrates and metals (gold, silver, and copper [1-5]), metal film morphologies and patterning methods aimed at creating maximally enhancing SERS surfaces. SERS, as many experiments have shown $[6,7]$, occurs due to the presence of a metal surface in proximity to the scattering molecule and contains two components; electromagnetic [8,9] and chemical [10-12]. Electromagnetic enhancement, the primary contribution to SERS is due to the excitation of a surface plasmon [8] (SP) which generates a strong, localized, secondary field, that Raman scatters from the molecules together with the incident field. These phenomena have been modeled for a single metal sphere [9] and a small illuminated metal particle $[13,14]$ and have also been extended to an array of spheres with varying sizes [15-17]. Experiments have revealed correlations between the SERS enhancement and the excited surface plasmon including works on tuning the surface plasmon resonance by different nanoparticles structures, sizes, and dielectric constants [18-23]. The models predict a Raman enhancement on gold or silver spheres of six to seven orders of magnitude and have been confirmed in experiments [24-27]. Specifically, the influence of gold and silver nanoparticle size in colloids $[3,4]$ and core shells [28] on the surface plasmon resonance and SERS enhancement have also been reported. It follows from the experiments and theoretical models [29] that the maximum SERS enhancements are observed when the SP resonance wavelength is equal to $\lambda_{\mathrm{sp}}=\left(\lambda_{\mathrm{exc}}+\lambda_{\mathrm{Rs}}\right) / 2$, where $\lambda_{\mathrm{sp}}, \lambda_{\mathrm{exc}}$, and $\lambda_{\mathrm{Rs}}$ are the surface plasmon, excitation, and Raman wavelength respectively. Furthermore, it was predicted that using different size of nanoparticles influences not only the surface plasmon wavelength but also the intensity of the electromagnetic field created in between the nanoparticles leading to higher SERS enhancements [15-17]. Differences between various metals were also discussed, revealing that the SERS enhancement of silver exceeds that of gold, which exceeds that of copper. The explanation for this lies in the parameter $\varepsilon_{b}$ (the contribution of interband transition to the dielectric function), when $\varepsilon_{b}$ is large, the width of the resonance increases and the SERS enhancement decreases [30]. 


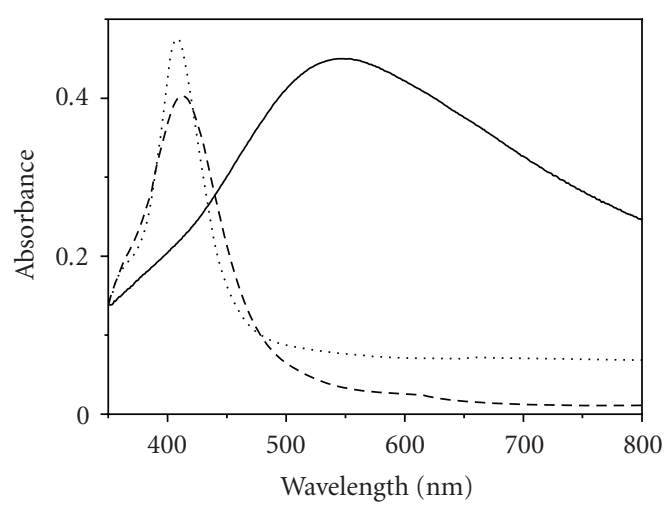

(a)

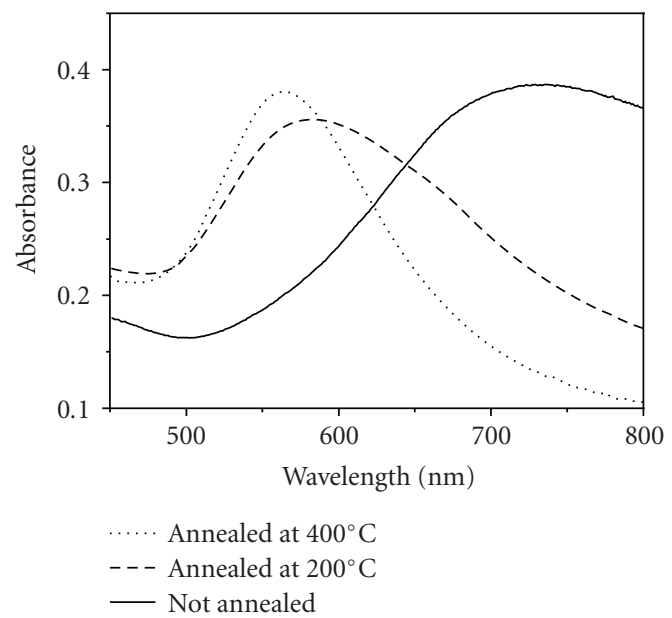

(b)

Figure 1: Absorption spectra of $\mathrm{Ag}$ (a) and $\mathrm{Au}$ (b) films of $\sim 10 \mathrm{~nm}$ thick annealed at different temperatures.

In this paper we examine the influence of different parameters (nanoparticle size, morphology, and composition as well as SP resonance wavelength) on SERS. We present an experimental study of SERS substrates consisting of gold, silver, and alloyed gold-silver nanoparticles of various sizes and compositions with the aim of finding the optimal conditions for fabricating substrates with maximum SERS enhancement factors. We have developed a simple technique of tuning the SP resonance frequency by a combination of vacuum deposition of silver and gold films with subsequent annealing of the deposited layers. We find that tuning by $\mathrm{Ag}$-Au alloying results in much smaller enhancement factors compared to tuning by deposition of small Ag nanoparticles over larger Au nanoparticles although the SP of Ag-Au alloy coincides better with the excitation wavelength. We show that a maximum Raman enhancement in the Ag-Au films can be obtained from an island film composed of Ag nanoparticles over Au particles, tuned to an SP resonance close to the excitation wavelength and possessing a double-peak size distribution in the particle ensemble.

\section{Experimental}

For substrates we use glass microscope slides, which were cleaned with soap and water, then rinsed with ethanol, and

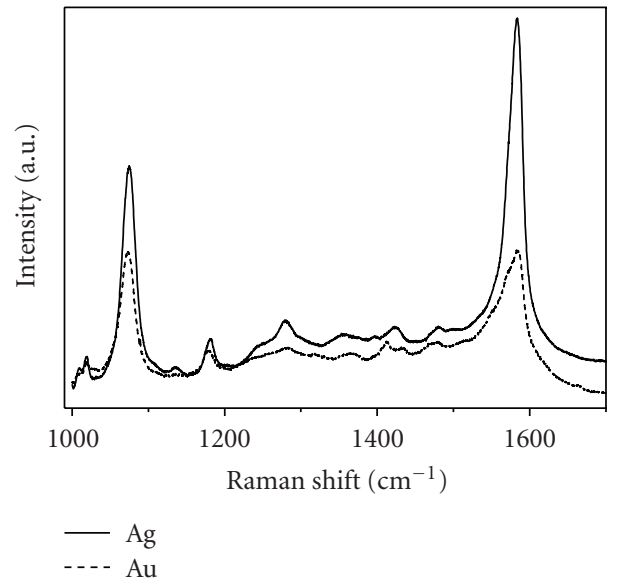

FIgURE 2: SERS spectra of MBA on silver (solid line) and gold (dashed line) films with $633 \mathrm{~nm}$ excitation wavelength. The film thicknesses of both films were $10 \mathrm{~nm}$; average particle sizes were $50 \mathrm{~nm}$.

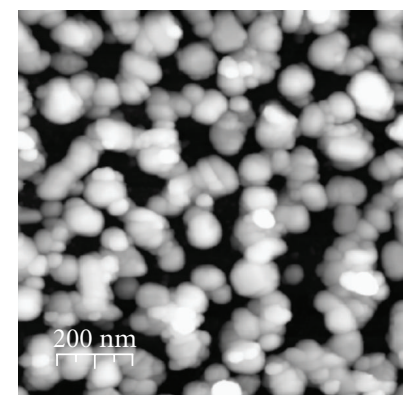

(a)

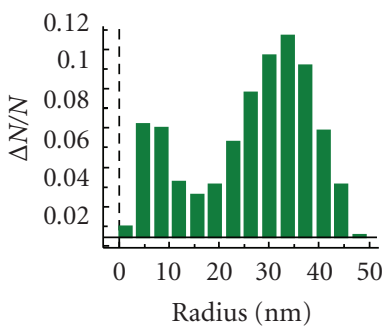

(c)

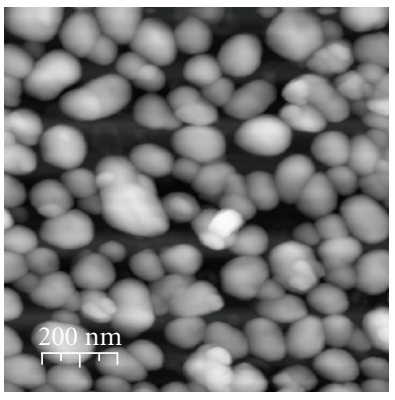

(b)

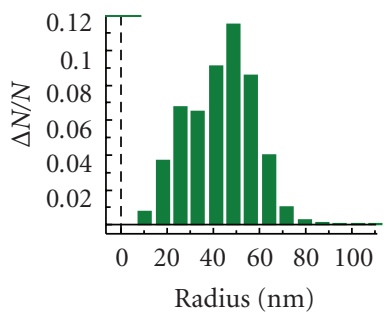

(d)
Figure 3: AFM scans and histograms of the two types of samples. (a) Annealed gold covered by annealed silver. (b) Gold covered by silver and annealed together. (c), (d) Histograms of the (a) and (b) samples, respectively.

dried with an air stream. Thin films of $\mathrm{Ag}$ and $\mathrm{Au}$, as well as two-layer thin films consisting of Ag over $\mathrm{Au}$ or Au over $\mathrm{Ag}$ were deposited by thermal evaporation in a vacuum of about $1 \cdot 10^{-6}$ Torr. In the case of two-layered films, after the deposition of the first layer (Au or Ag of 5-15 nm thickness) some of the samples were annealed in hydrogen in the range $200-400^{\circ} \mathrm{C}$ for various times. Then the second layer of 2-10 nm thick was evaporated over the first layer (Ag over Au or Au over Ag). After deposition of the second layer the samples were annealed in hydrogen at $200-400^{\circ} \mathrm{C}$ for 


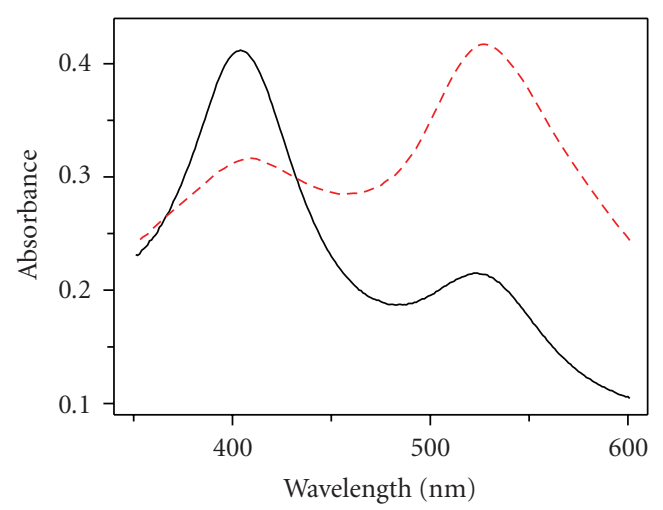

(a)

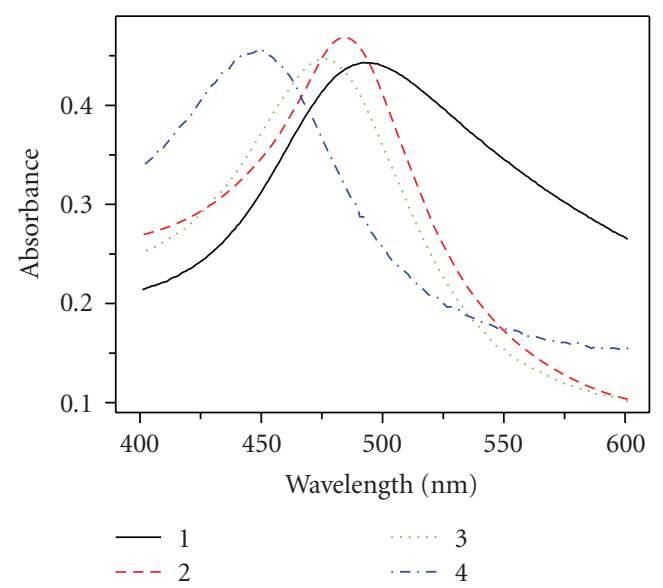

(b)

FIgURE 4: Absorption spectra of the two types of samples, with various metal concentrations. (a) Annealed gold covered by annealed silver (samples A), 1, 2: various silver concentrations; $~ 80$, $20 \%$, respectively. (b) Gold covered by silver and annealed together (samples B), 1-4: various silver concentrations; 20\%, 40\%, 50\%, and $80 \%$, respectively.

various times. The annealing process was done in hydrogen in order to avoid oxidation of the silver layer. The films were characterized by their optical absorption spectrum, and the film morphologies were examined using an atomic force microscope (AFM). For SERS measurements the substrates with the annealed films were spin coated with a homogenous layer of $6 \mathrm{mM}$ of 4-mercaptobenzoic acid (4-MBA) dissolved in ethanol. According to our estimates, after the ethanol evaporation, the effective thickness of 4-MBA layer covering the nanoparticles was about $0.3 \mathrm{~nm}$. SERS measurements were obtained in a Jobin Yvon HR800 Raman microscope with $514 \mathrm{~nm}$ and $633 \mathrm{~nm}$ excitation lasers with incident intensities of $1 \times 10^{5}$ and $2.5 \times 10^{5} \mathrm{~W} / \mathrm{cm}^{2}$, respectively.

\section{Results and Discussion}

Examination by AFM showed that after deposition, both the Ag and Au films had a granular (island) structure. Annealing of the films causes a surface mass transfer between the

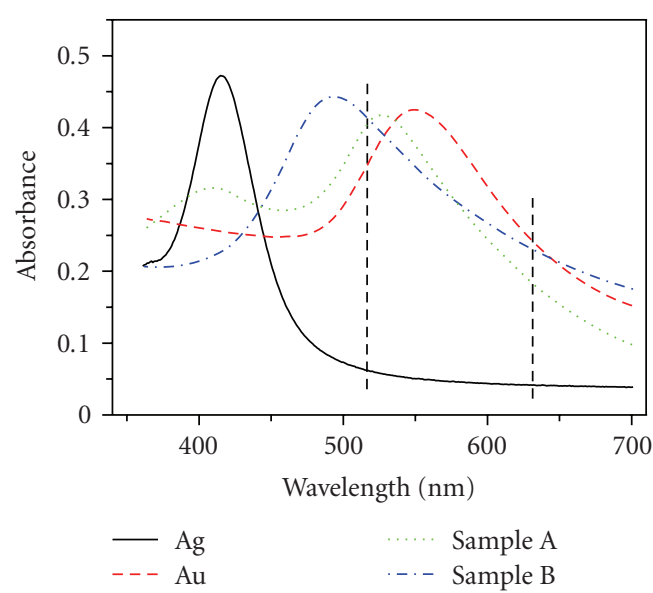

FIGURE 5: Absorption spectra of the two types of samples and pure gold and silver annealed at $400^{\circ} \mathrm{C}$ and $200^{\circ} \mathrm{C}$, resp.

islands as well as alloying in two-layered films due to bulk interdiffusion at the annealing temperature. Surface mass transfer between the islands was noticeable at $200^{\circ} \mathrm{C}$ for $\mathrm{Ag}$ and at $400^{\circ} \mathrm{C}$ for $\mathrm{Au}$ and resulted in the formation of equilibrium nanoparticle shape close to a spherical segment with the wetting angle of about 80 degrees, both for $\mathrm{Ag}$ and $\mathrm{Au}$. We also observed sintering and agglomeration of nanoparticles located near one another and diffusion coalescence of nanoparticles $[31,32]$ resulting in an increase of the average particle size. Due to the transformation of the random nanoparticle shapes to nearly spherical and the increase of the average particle size, SP resonance shifts to the blue and becomes narrower (Figure 1), in good agreement with previous studies [33].

Comapring the SERS enhancement from Ag and Au films (Figure 2) showed that the enhancement factor obtained with the Ag film was several times higher than that for the Au film, even for an excitation wavelength of $633 \mathrm{~nm}$, which is closer to SP resonance of $\mathrm{Au}$. This result is in a good agreement with theoretical predictions [30, 34] in which silver nanoparticles show a higher SERS enhancement than gold. On this basis we studied two-layered films, in which the Ag layer was deposited over the gold layer.

In the preparation of nanoclusters consisting of both $\mathrm{Ag}$ and $\mathrm{Au}$ we examined two different annealing procedures, by which we created two types of substrates. In the first (sample A) we created the two-layered films in two stages; deposition of $\mathrm{Au}$, annealing at $400^{\circ} \mathrm{C}$, and deposition of $\mathrm{Ag}$ and then annealing at $200^{\circ} \mathrm{C}$. The particle size distributions of such samples, measured by AFM, show two peaks (Figure 3(c)). The additional peak at $R \approx 5 \mathrm{~nm}$ is referred to the $\mathrm{Ag}$ nanoparticles formed after the second annealing (at $200^{\circ} \mathrm{C}$ ) which are smaller than the gold particles formed during the first annealing at $400^{\circ} \mathrm{C}$. The optical absorption spectra also shows two SP resonances: one is near the Au SP (with some shift to the blue) and the second near the Ag SP. The position of the SP resonances depends on the concentration of each metal and the annealing time at $200^{\circ} \mathrm{C}$ (Figure $4(\mathrm{a})$ ). The fact that we observe two separate plasmon resonances implies 


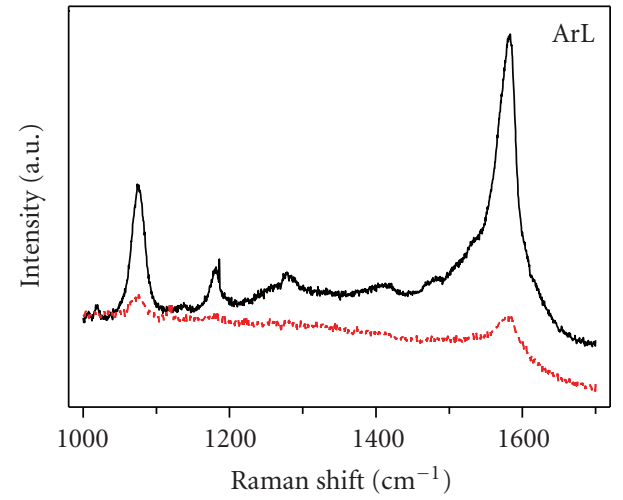

(a)

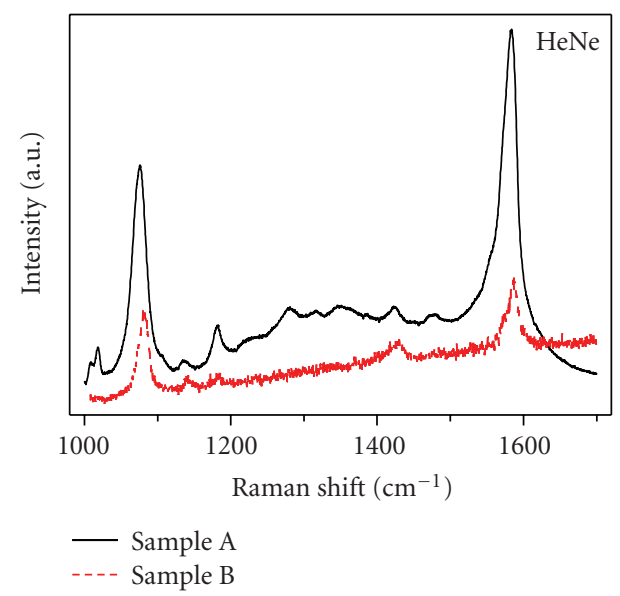

(b)

Figure 6: SERS measurements of 4-MBA on the two different substrates (sample A-black solid line, sample B-red dashed line) using two excitation lasers, $514 \mathrm{~nm}(\mathrm{ArL})$ and $633 \mathrm{~nm}(\mathrm{HeNe})$ left and right graph, resp.

that annealing at $200^{\circ} \mathrm{C}$ leaves the silver and gold nanoparticles compositionally separated, although each becomes sintered. Indeed, the Ag diffusion coefficient into gold at $200^{\circ} \mathrm{C}$ is $2.6 \times 10^{-24} \mathrm{~m}^{2} / \mathrm{s}$, and the interdiffusion of the two metals would require an incredibly long time to form even a very thin alloyed layer (about 670 hours to form a $5 \mathrm{~nm}$ layer).

In the second annealing procedure (sample B) we annealed two layered gold-silver films at $400^{\circ} \mathrm{C}$. In this process we obtained bulk interdiffusion between the silver and gold and the annealing produced homogeneous islands (Figure 3(b)) with a normal size distribution (Figure 3(d)) and an SP resonance located between the SP of pure Ag and pure Au films (Figure 4(b)), with exact position depending on the concentration of each metal. Thus, annealing at $400^{\circ} \mathrm{C}$ results in formation of alloyed nanoparticles due to diffusion of silver into gold. The diffusion of silver into gold is faster than gold diffusion into silver [35], and at $400^{\circ} \mathrm{C}$ the diffusion coefficient is about $D=8 \times 10^{-19} \mathrm{~m}^{2} / \mathrm{s}$, that is, the width of diffusion zone $2(D t)^{1 / 2}$ (the thickness of the alloyed layer in Au nanoparticles) becomes equal to the particle radius $(R=50 \mathrm{~nm})$ for a time $t \sim 15$ minutes; several times shorter than the annealing time in our experiments (12 hours depending on the film thickness).

To demonstrate how the morphology of Au-Ag films affects the SERS enhancement, we present SERS spectra obtained from both types A and B substrates.

Spectral absorption measurements of the type A and B substrates as well as pure silver (annealed at $200^{\circ} \mathrm{C}$ ) and pure gold (annealed at $400^{\circ} \mathrm{C}$ ) are shown in Figure 5 (the two vertical dashed lines mark the two laser excitation wavelengths (514 and $633 \mathrm{~nm}$ ) used for SERS measurements). As shown in Figure 5 there are two absorption peaks for sample A, at 528 and $408 \mathrm{~nm}$, while for sample B a single peak at $494 \mathrm{~nm}$ is observed. The pure gold and silver films have absorption peaks at $550 \mathrm{~nm}$ and $415 \mathrm{~nm}$, resp.

Based only on the proximity of the laser excitation wavelength at $514 \mathrm{~nm}$ to the plasmon resonance we would expect both samples to provide good enhancement with a small advantage for substrate B which has a single strong plasmon resonance slightly blue shifted from the laser line [29]. The SERS measurements performed on 4-MBA, however, reveal that substrate A provides higher enhancement at both excitation wavelengths $(514 \mathrm{~nm}$ and $633 \mathrm{~nm}$ ) as shown in Figure 6.

These results clearly demonstrate that SERS enhancement depends not only on the proximity of the excitation wavelength to the plasmon peak position but also on the size distribution of the nanoparticles and the metal type. Since silver is known to be a better enhancing metal for SERS [29] and as we claimed above, in samples A, we have a silver layer on top of the SERS substrate while in samples B we have an alloy of gold and silver, it is reasonable that sample A should have the better performance. A further advantage of the twolayered samples (sample A) is that the method of preparation results in a wider cluster size distribution. Since it has been observed experimentally that the SERS enhancement occurs at specific "hot spots" on the surface, and based on theory [15-17] these "hot spots" are due to specifically sized cluster lying in close proximity to each other, a broader size distribution is expected to lead to a higher probability to find such cluster sizes near each other and thus to increased enhancement. Another advantage of the two-layered substrate is its broader, two-peaked plasmon resonance, which allows for a broader choice for the excitation wavelength.

\section{Conclusion}

We compare between two ways of creating Ag-Au SERS substrates, resulting in alloyed and two-layered films. We show that the surface plasmon resonance wavelength can be tuned by the alloying composition of the nanoparticles and the annealing process whereby we can create a film with one or two plasmon peaks. We demonstrate that the technique resulting in a two-layered film, in which small silver nanoparticles lie on top of larger gold nano particles, results in a better SERS surfaces that shows significantly higher Raman enhancements than alloyed gold-silver nano particles.

\section{Acknowledgment}

This work was supported by the Israel Ministry of Defense. 


\section{References}

[1] T. Wang, X. Hu, and S. Dong, "Surfactantless synthesis of multiple shapes of gold nanostructures and their shapedependent SERS spectroscopy," Journal of Physical Chemistry B, vol. 110, no. 34, pp. 16930-16936, 2006.

[2] S. P. Mulvaney, L. He, M. J. Natan, and C. D. Keating, "Three-layer substrates for surface-enhanced Raman scattering: preparation and preliminary evaluation," Journal of Raman Spectroscopy, vol. 34, no. 2, pp. 163-171, 2003.

[3] L. Rivas, S. Sanchez-Cortes, J. V. Garcia-Ramos, and G. Morcillo, "Mixed silver/gold colloids: a study of their formation, morphology, and surface-enhanced Raman activity," Langmuir, vol. 16, no. 25, pp. 9722-9728, 2000.

[4] F. Jinghuai, H. Yunxis, L. Xia, and D. Xiaoming, "Aggregation and surface-enhanced Raman activity study of dye-coated mixed silver-gold colloids," Journal of Raman Spectroscopy, vol. 35, p. 914, 2004.

[5] N. R. Jana, "Silver coated gold nanoparticles as new surface enhanced Raman substrate at low analyte concentration," The Analyst, vol. 128, no. 7, pp. 954-956, 2003.

[6] M. G. Albrecht, J. F. Evans, and J. A. Creighton, "The nature of an electrochemically roughened silver surface and its role in promoting anomalous Raman scattering intensity," Surface Science, vol. 75, no. 4, pp. L777-L780, 1978.

[7] D. L. Jeanmaire and R. P. Van Duyne, "Surface Raman spectroelectrochemistry-part I: heterocyclic, aromatic, and aliphatic amines adsorbed on the anodized silver electrode," Journal of Electroanalytical Chemistry, vol. 84, no. 1, pp. 1-20, 1977.

[8] M. Moskovits, "Surface-enhanced spectroscopy," Reviews of Modern Physics, vol. 57, no. 3, pp. 783-826, 1985.

[9] G. C. Schatz and R. P. Van Duyne, Handbook of Vibrational Spectroscopy, 2002.

[10] B. N. J. Persson, "On the theory of surface-enhanced Raman scattering," Chemical Physics Letters, vol. 82, no. 3, pp. 561565,1981 .

[11] P. Kambhampati, C. M. Child, M. C. Foster, and A. Campion, "On the chemical mechanism of surface enhanced Raman scattering: experiment and theory," The Journal of Chemical Physics, vol. 108, no. 12, pp. 5013-5026, 1998.

[12] J. R. Lombardi, R. L. Birke, T. Lu, and J. Xu, "Charge-transfer theory of surface enhanced Raman spectroscopy: HerzbergTeller contributions," The Journal of Chemical Physics, vol. 84, no. 8, pp. 4174-4180, 1986.

[13] D.-S. Wang, M. Kerker, and H. W. Chew, "Raman and fluorescent scattering by molecules embedded in dielectric spheroids," Applied Optics, vol. 19, no. 14, pp. 2315-2328, 1980.

[14] D.-S. Wang and M. Kerker, "Enhanced Raman scattering by molecules adsorbed at the surface of colloidal spheroids," Physical Review B, vol. 24, no. 4, pp. 1777-1790, 1981.

[15] K. Li, X. Li, M. I. Stockman, and D. J. Bergman, "Surface plasmon amplification by stimulated emission in nanolenses," Physical Review B, vol. 71, no. 11, Article ID 115409, 5 pages, 2005.

[16] K. Li, M. I. Stockman, and D. J. Bergman, "Enhanced second harmonic generation in a self-similar chain of metal nanospheres," Physical Review B, vol. 72, no. 15, Article ID 153401, 4 pages, 2005.

[17] K. Li, M. I. Stockman, and D. J. Bergman, "Self-similar chain of metal nanospheres as an efficient nanolens," Physical Review Letters, vol. 91, no. 22, Article ID 227402, 4 pages, 2003.
[18] C. L. Haynes and R. P. Van Duyne, "Plasmon-sampled surfaceenhanced Raman excitation spectroscopy," Journal of Physical Chemistry B, vol. 107, no. 30, pp. 7426-7433, 2003.

[19] R. D. Averitt, D. Sarkar, and N. J. Halas, "Plasmon resonance shifts of Au-coated Au2S nanoshells: insight into multicomponent nanoparticle growth," Physical Review Letters, vol. 78, no. 22, pp. 4217-4220, 1997.

[20] T. R. Jensen, M. D. Malinsky, C. L. Haynes, and R. P. Van Duyne, "Nanosphere lithography: tunable localized surface plasmon resonance spectra of silver nanoparticles," Journal of Physical Chemistry B, vol. 104, no. 45, pp. 10549-10556, 2000.

[21] C. L. Haynes, A. D. McFarland, L. Zhao, et al., "Nanoparticle optics: the importance of radiative dipole coupling in twodimensional nanoparticle arrays," Journal of Physical Chemistry B, vol. 107, no. 30, pp. 7337-7342, 2003.

[22] N. M. B. Perney, F. J. G. de Abajo, J. J. Baumberg, et al., "Tuning localized plasmon cavities for optimized surface-enhanced Raman scattering," Physical Review B, vol. 76, no. 3, Article ID 035426, 2007.

[23] J. Grand, M. L. de La Chapelle, J.-L. Bijeon, P.-M. Adam, A. Vial, and P. Royer, "Role of localized surface plasmons in surface-enhanced Raman scattering of shape-controlled metallic particles in regular arrays," Physical Review B, vol. 72, no. 3, Article ID 033407, 4 pages, 2005.

[24] P. W. Barber, R. K. Chang, and H. Massoudi, "Electrodynamic calculations of the surface-enhanced electric intensities on large Ag spheroids," Physical Review B, vol. 27, no. 12, pp. 7251-7261, 1983.

[25] F. J. Garcia-Vidal and J. B. Pendry, "Collective theory for surface enhanced Raman scattering," Physical Review Letters, vol. 77, no. 6, pp. 1163-1166, 1996.

[26] J. Gersten and A. Nitzan, "Radiative properties of solvated molecules in dielectric clusters and small particles," The Journal of Chemical Physics, vol. 95, no. 1, pp. 686-699, 1991.

[27] M. Kerker, D.-S. Wang, and H. Chew, "Surface enhanced Raman scattering (SERS) by molecules adsorbed at spherical particles," Applied Optics, vol. 19, no. 19, pp. 3373-3388, 1980.

[28] M. Mandal, N. R. Jana, S. Kundu, S. K. Ghosh, M. Panigrahi, and T. Pal, "Synthesis of Aucore-Agshell type bimetallic nanoparticles for single molecule detection in solution by SERS method," Journal of Nanoparticle Research, vol. 6, no. 1, pp. 53-61, 2004.

[29] N. Félidj, J. Aubard, G. Lévi, et al., "Controlling the optical response of regular arrays of gold particles for surfaceenhanced Raman scattering," Physical Review B, vol. 65, no. 7, Article ID 075419, 9 pages, 2002.

[30] K. Kneipp, M. Moskovits, and H. Kneipp, Surface-Enhanced Raman Scattering: Physics and Applications, Springer, Berlin, Germany, 2006.

[31] I. M. Lifshitz and V. V. Slyozov, "The kinetics of precipitation from supersaturated solid solutions," Journal of Physics and Chemistry of Solids, vol. 19, no. 1-2, pp. 35-50, 1961.

[32] Yu. Kaganovskii and L. N. Paritskaya, "Diffusion in Nanomaterials," Encylopedia of Nanoscience and Nanotechnology, vol. 2, p. 399, 2004.

[33] R. Gupta, M. J. Dyer, and W. A. Weimer, "Preparation and characterization of surface plasmon resonance tunable gold and silver films," Journal of Applied Physics, vol. 92, no. 9, p. 5264, 2002.

[34] E. J. Zeman and G. C. Schatz, "An accurate electromagnetic theory study of surface enhancement factors for $\mathrm{Ag}, \mathrm{Au}, \mathrm{Cu}$, Li, Na, Al, Ga, In, Zn, and Cd," Journal of Physical Chemistry, vol. 91, no. 3, pp. 634-643, 1987.

[35] W. C. Mallard, A. B. Gardner, R. F. Bass, and L. M. Slifkin, "Self-diffusion in silver-gold solid solutions," Physical Review, vol. 129, no. 2, pp. 617-625, 1963. 

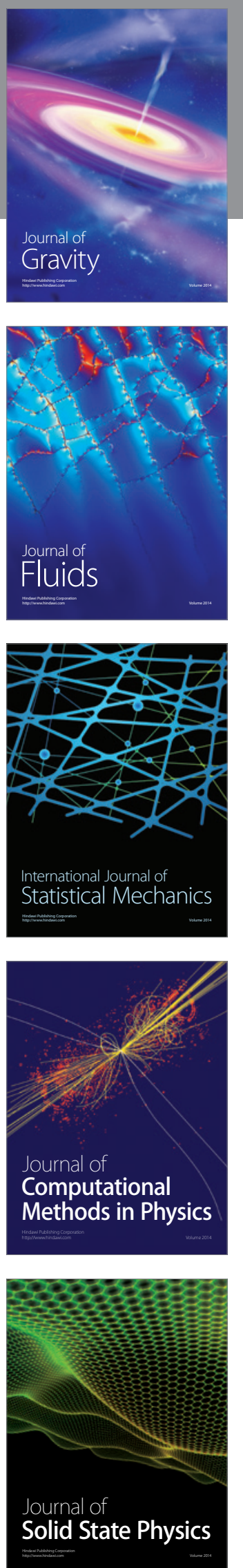

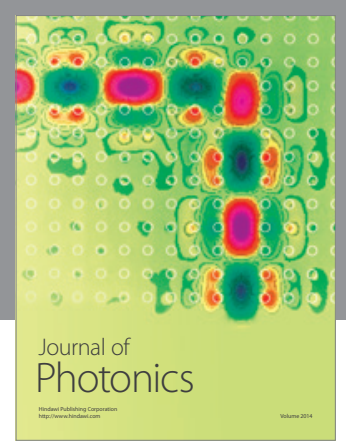

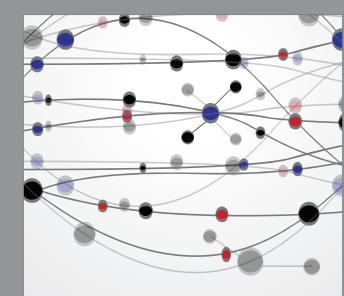

The Scientific World Journal
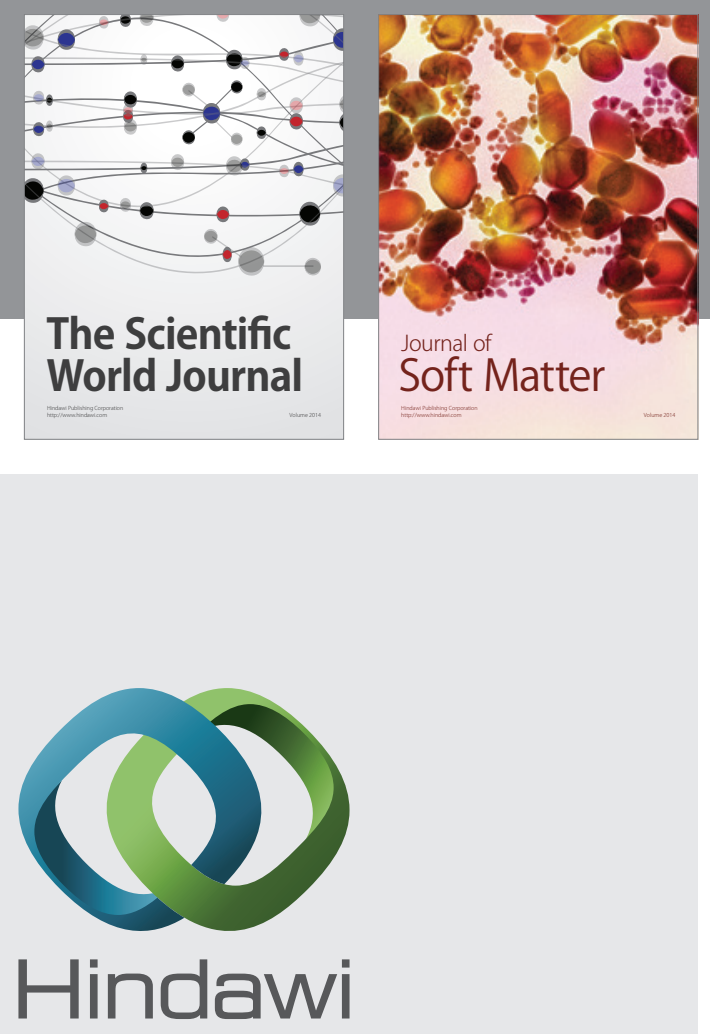

Submit your manuscripts at

http://www.hindawi.com
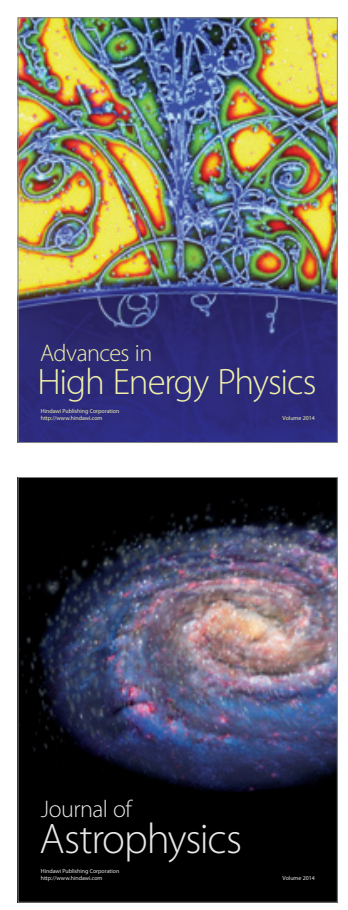
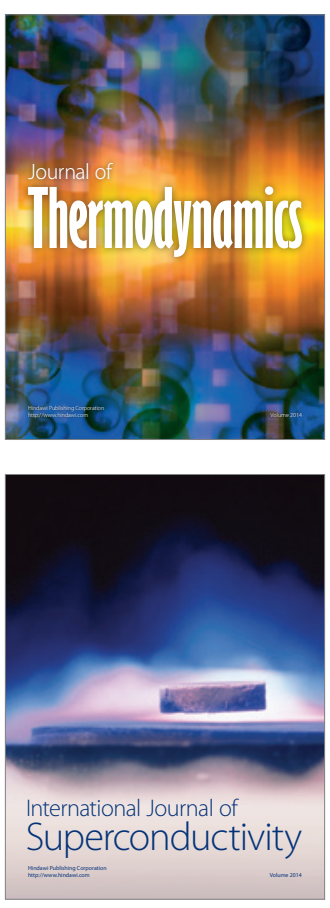
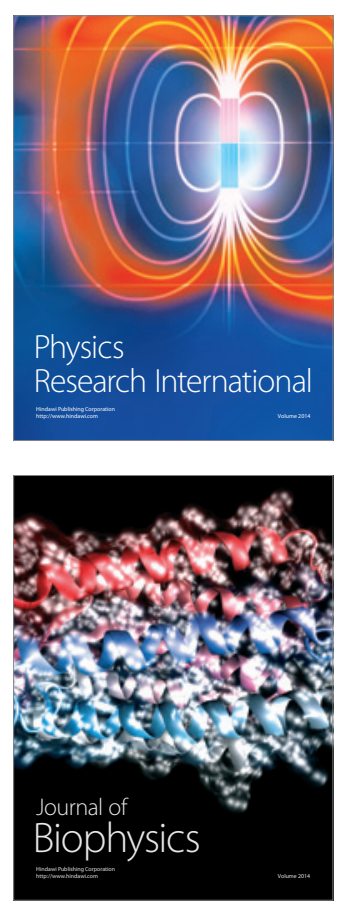
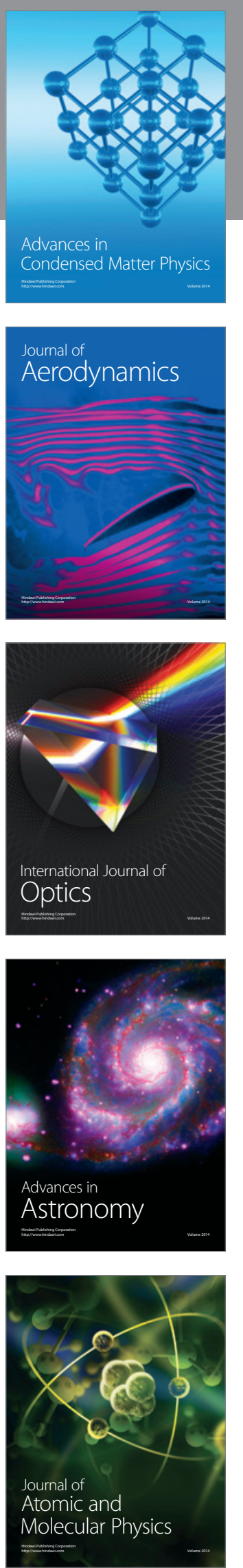